\title{
Evaluación ex - post del proyecto construcción represa y sistema de riego cuartos en Bolivia
}

\author{
Ex-post evaluation of the project construction dam and irrigation \\ system rooms in Bolivia
}

\author{
María Angélica Alizares Jaén \\ malizaresjaen@gmail.com \\ Universidad Autónoma Tomás Frías, Bolivia
}

Artículo recibido enero 2019 | Arbitrado en feb-marzo 2019 | Publicado en mayo 2019

\section{RESUMEN}

La evaluación permite conocer la realidad del funcionamiento $\mathrm{y}$ resultados del proyecto ya ejecutado y puesto en funcionamiento, luego de que el proyecto inicia con la operatividad, se puede visualizar cambios y mejoras en la producción, pero también deficiencias y el incumplimiento de metas propuestas, ya que se debe tener en cuenta que fue el Estado boliviano a través del Gobierno Autónomo Departamental de Potosí quien invirtió un presupuesto para la ejecución del proyecto, por lo tanto los resultados deben ser óptimos. La Evaluación Ex-post del Proyecto "Represa y Sistema de Riego Cuartos", evalúa aspectos que se tomaron en cuenta para la formulación del Proyecto de Inversión Pública con la que fue aprobado. Se plantea como objetivo realizar la evaluación Ex - Post del Proyecto Represa y Sistema de Riego Cuartos, en base a los resultados de conocer los criterios de evaluación. Para la evaluación Ex - Post del proyecto Represa y Sistema de Riego Cuartos, se aplicó la guía publicada por el Ministerio de Medio Ambiente y Aguas el 2014, el cual permitió conocer las mejoras a la calidad de vida de los beneficiarios. El resultado final de la evaluación Ex-post de resultados logra una calificación del 61\%, muestra que el proyecto alcanzo un rango de Medio y los resultados son similares a lo esperado. El resultado final de la evaluación Ex-post de efectos logra una calificación del $41 \%$, muestra que el proyecto alcanzo un rango de Medio y los resultados son similares a lo esperado.

Palabras clave: Evaluación ex - post; proyecto; represas, sistema de riegos

\begin{abstract}
The evaluation allows to know the reality of the operation and results of the project already executed and put into operation, after the project starts with the operation, changes and improvements in production can be visualized, but also deficiencies and the non-fulfillment of proposed goals, already It must be taken into account that it was the Bolivian State through the Autonomous Departmental Government of Potosí that invested a budget for the execution of the project, therefore the results must be optimal. The Ex-post Evaluation of the Project "Dam and Room Irrigation System", evaluates aspects that were taken into account for the formulation of the Public Investment Project with which it was approved. The objective is to carry out the Ex Post evaluation of the Dam and Room Irrigation System Project, based on the results of knowing the evaluation criteria. For the Ex - Post evaluation of the Dam and Room Irrigation System project, the guide published by the Ministry of Environment and Water in 2014 was applied, which allowed to know the improvements to the quality of life of the beneficiaries. The final result of the Ex-post evaluation of results achieves a rating of $61 \%$, showing that the project reached a Medium range and the results are similar to expectations. The final result of the Ex-post evaluation of effects achieves a rating of $41 \%$, showing that the project reached a Medium range and the results are similar to what was expected.
\end{abstract}

Key words: Ex-post evaluation; draft; dams, irrigation system 


\section{INTRODUCCIÓN}

En nuestro medio, las experiencias en evaluación Ex-post de proyectos son muy limitados y la escases de documentos que señalen si existe impacto de las inversiones del Gobierno, es decir, se desconoce si la población asentada en las área de influencia de dicha inversión ha experimentado incremento alguno en el bienestar de la población, así también no se dispone de información publicada a nivel Ex-post que permita retroalimentar las etapas tanto de la pre inversión como de la inversión por lo que el presente trabajo de investigación constituye un tema de vital importancia desde el punto de vista científico.

La falta de certeza de las estimaciones de los efectos e impactos de los proyectos, conduce a la búsqueda de sistemas en el cual exista una comparación entre lo previsto y lo real, permitan por un lado mejorar los sistemas y metodologías de evaluación Ex Ante, y por otro, realizar correcciones al proyecto durante su ejecución u operación, a fin de evitar la evolución hacia resultados desfavorables. Por último, debe generarse todo un mecanismo de retroalimentación del proceso de inversión que garantice ir mejorando cada vez más los resultados obtenidos.

Por tanto, en la comunidad de Cuartos se ha ejecutado el proyecto "Construcción Represa y Sistema de Riego Cuartos", por lo que la población asentada en la zona depende básicamente de actividades agrícola a temporal y pecuaria, al permitir desarrollar actividades como la agricultura y la ganadería (principales fuentes de ingreso). En ese sentido, buscando estos objetivos que se suponen deben cumplir los proyectos de riego, se han ejecutado numerosos proyectos. Sin embargo, sus resultados no son alentadores, lo que hace pensar que algo no está funcionando bien.
Esta realidad refleja el poco interés que existe por parte de los usuarios en la conservación y en el mantenimiento de las infraestructuras de riego que se suponen es para desarrollar sus actividades agrícolas. Se formulan proyectos basados generalmente en modelos, que son ajenos a esta realidad. En este sentido la evaluación Ex-post, de la Represa y Sistema de Riego Cuartos, permitirá conocer los niveles de los impactos, la eficacia, eficiencia, la pertinencia y sostenibilidad del proyecto.

Es ese entender, la evaluación Ex-post en la Represa y Sistema de Riego Cuartos, se orientará a una verdadera y permanente participación del poblador, predominando actitudes de reflexión decisión y responsabilidad sobre un desarrollo coherente, basado en demandas rurales donde la promoción, capacitación y la evaluación formen parte del que hacer institucional.

A efectos de verificar que la población beneficiaria de dicho proyecto de irrigación está recibiendo los servicios en la cantidad y con la calidad prevista, así como obtener lecciones aprendidas sobre los procesos que se ha seguido en cada fase del ciclo de éstos, se requiere evaluar la situación en la que se encuentra cada uno y contrastarla con las previsiones que se realizaron en los estudios de pre inversión.

En el marco del (PND) Plan Nacional de Desarrollo, en sus atribuciones del Ministerio de Medio Ambiente y Agua ha elaborado para el 2014 la guía para la evaluación Ex - Post de proyectos de riego porque considera de imperiosa necesidad la evaluación los proyectos de riego ejecutados por todas instituciones ejecutoras de proyectos de inversión pública; con el propósito de analizar y evaluar el cumplimiento de los indicadores de 
desempeño sobre los efectos logrados con las inversiones en los sistemas de riego.

Se aplicará la guía de evaluación Ex Post de proyecto de Riego (2015) propuesta por el Ministerio de Medio Ambiente y Aguas para determinar la evaluación ex - post de resultados y la evaluación ex - post de efectos del proyecto ejecutado.

El enfoque práctico del documento pretende ser una guía didáctica de fácil manejo por parte de los operadores y administradores de proyectos, por último, se muestran las pautas básicas de aplicación de la metodología y el tipo de información requerida para articular el ciclo de vida de los proyectos con la evaluación expost.

El Campos de acción de la investigación es la Evaluación Ex - Post de proyectos de riego.

\section{Antecedentes históricos}

En el país, hasta el momento no se dispone de método alguno para realizar una evaluación de resultados y de efectos de los proyectos de riego que están en funcionamiento. La evaluación que se efectúa es en situaciones esporádicas. Para el 2014 el Ministerio de Medio Ambiente y Aguas propuso una guía metodológica para la evaluación Ex - Post de proyectos de riego, pero no se tiene experiencia alguna debido a que las evaluaciones se realizan años después de su ejecución.

A pesar de los miles de millones de bolivianos que el Estado emplea para tratar de coadyuvar con el desarrollo del país, aún se desconoce el efecto real de cada uno de los proyectos que han sido ejecutados y que se encuentran en pleno funcionamiento (Montaño, Rodríguez, Panche, y Sosa, s.f.).

Muchos gobiernos, instituciones $\mathrm{y}$ administradores de proyectos de nuestro medio se muestran evasivos a realizar evaluaciones Ex-post, porque las consideran costosas, prolongadas y técnicamente complejas y porque los resultados pueden ser políticamente delicados, especialmente si son negativos (Montaño, Rodríguez, Panche, y Sosa, s.f.).

El año 2000 se publicó el "Inventario Nacional de Sistemas de Riego", contribuyendo al desarrollo del sector con valiosa información sobre la situación del riego en Bolivia y la posibilidad de utilizarla para la planificación y dimensionamiento de futuras inversiones (MMAYA, 2013).

Doce años después, el Ministerio de Medio Ambiente y Agua, a través del Viceministerio de Recursos Hídricos y Riego, con el apoyo del Programa de Desarrollo Agropecuario Sustentable de la Cooperación Sueca y Alemana (PROAGRO/GIZ), ha efectuado la actualización de ese inventario para conocer la dimensión de los cambios alcanzados en la agricultura bajo riego en Bolivia y llevar a cabo una revisión de los resultados de las inversiones que han sido realizadas, durante más de una década, en la implementación de proyectos de riego en las zonas áridas y semiáridas del país (MMAYA, 2013), los detalle se muestran en tabla siguiente. (Tabla 1). 
Tabla 1. Incremento de los Sistemas de Riego por Departamento (2000-2012)

\begin{tabular}{|c|c|c|c|c|c|c|c|c|}
\hline \multirow[t]{2}{*}{ Departamento } & \multicolumn{2}{|c|}{ Sistemas de Riego } & \multicolumn{3}{|c|}{ Familias Regantes } & \multicolumn{3}{|c|}{ Área Regada Año (ha) } \\
\hline & $2000 \quad 2012$ & Incremento & 2000 & 2012 & Incremento & 2000 & 2012 & Incremento \\
\hline Chuquisaca & $678 \quad 746$ & 68 & 17,718 & 21,071 & 3,353 & 21,168 & 29,721 & 8,553 \\
\hline Cochabamba & $1,0351,333$ & 298 & 81,9251 & 112,223 & 30,298 & 87,534 & 95,950 & 8,416 \\
\hline La Paz & 9611,072 & 111 & 54,618 & 64,969 & 10,351 & 35,993 & 54,002 & 18,009 \\
\hline Oruro & $312 \quad 469$ & 157 & 9,934 & 16,288 & 6,354 & 14,039 & 18,442 & 4,403 \\
\hline Potosí & 9561,068 & 112 & 31,940 & 36,567 & 4,627 & 16,240 & 27,785 & 11,545 \\
\hline Santa Cruz & $232 \quad 306$ & 74 & 5,865 & 9,663 & 3,798 & 15,239 & 31,645 & 16,406 \\
\hline Tarija & $550 \quad 675$ & 125 & 15,975 & 22,646 & 6,671 & 36,351 & 45,656 & 9,305 \\
\hline TOTAL: & $4,7245,669$ & 945 & 217,9752 & 83,427 & 65,4522 & 226,5643 & 303,201 & 76,637 \\
\hline
\end{tabular}

Sistema de información de Riego (Fuente: VRHR-PROAGRO, MMAYA, 2012)

Si bien se actualizo el inventario, pero no se realizó la evaluación Ex - Post de los proyectos para ver los efectos e impactos de los proyectos concluidos (Gobierno Autónomo Departamental de Potosí, 2016).

El Ministerio de Medio Ambiente y Agua, junto al Viceministerio de Recursos Hídricos y Riego, público en abril de la gestión 2014 una herramienta denominado Guía para la Evaluación Ex-Post de Proyectos de Riego, debido a que el Ministerio de Medio Ambiente y Agua, considera de imperiosa necesidad la evaluación los proyectos de riego ejecutados por todas instituciones ejecutoras de proyectos de inversión pública; con el propósito de analizar y evaluar el cumplimiento de los indicadores de desempeño sobre los efectos logrados con las inversiones en los sistemas de riego (MMAYA, 2014).

El año 2016 de acuerdo al Convenio Interinstitucional 024/2016, entre la Dirección Ejecutiva del SENARI y el Ministro de Medio Ambiente de Agua, logran consolidar la modalidad de ejecución compartida SENARI- UCP CAF (2014) del Programa MIAGUA III, en el componente de A/AT. Entonces la UCP CAF (2014) asume la responsabilidad administrativa financiera para la ejecución del componente A/AT y el SENARI asume la responsabilizada Técnica para la ejecución del componente A/AT (componente 1,6 "Supervisión a la Asistencia Técnica Integral").

El diagnóstico y evaluación de proyectos Ex-post A/AT, realizada en abril hasta noviembre del 2016 tuvieron los siguientes resultados teniendo el siguiente diagnóstico de la evaluación, de los 32 proyectos que se muestran en la tabla 2 que representan el $100 \%$ de los proyectos reportados con diagnósticos funcionan un $94 \%$ necesitan apoyo en gestión, infraestructura y producción indistintamente y un $6 \%$ no funcionan y también necesitan apoyo en gestión, infraestructura y producción (Almendras y Beltrán, 2016). 
Tabla 2. Requerimiento de Apoyo Ex-post 32 Proyectos

\begin{tabular}{clll}
\hline Cat. & \multicolumn{1}{c}{ Valoración } & N Proy. POTOSí & $\mathbf{9}$ \\
\hline 1 & SR. Desistido & 0 & 0 \\
2 & El SR. Está en Funcionamiento & 3 & 10 \\
3 & F/ Necesita Apoy. Solo Gestión & 11 & 35 \\
4 & F/ Necesita Apoy. En Gestión e Infraest. & 2 & 6 \\
5 & F/ Necesita Apoy. En Gestión y Prod. & 9 & 28 \\
6 & F/ Necesita Apoy. En Gestión, Infraest y Prod. & 2 & 6 \\
7 & F/ Necesita Apoy. En Solo Prod. & 0 & 0 \\
8 & F/ Necesita Apoy. Solo En Infraest. & 3 & 9 \\
9 & El SR. NO Está en Funcionamiento A/ G.I. & 1 & 3 \\
10 & El SR. NO Está en Funcionamiento A/ G.I. P. & 1 & 3 \\
& $\quad$ TOTAL & $\mathbf{3 2}$ & $\mathbf{1 0 0}$ \\
\hline
\end{tabular}

Requerimiento de apoyo Ex-post (Fuente: INFORME DE LOS ING. ALMENDRAS, BELTRAN, 2016)

El proyecto "Construcción Represa y Sistema de Riego Cuartos", ha sido concluido y entregado definitivamente a los beneficiarios en fecha 30 de agosto del 2016 y los ejecutores del mismo dieron por concluido su intervención. Sin embargo, se desconoce los efectos e impactos del proyecto concluido, además se tiene información de la evaluación del grado de cumplimiento de los objetivos y metas del proyecto propuestos con los objetivos y metas del proyecto alcanzados (Gobierno Autónomo Departamental De Potosí, 2016).

En el siguiente trabajo se darán a conocer diversos temas de interés sobre la evaluación Ex-post de proyectos de riego, tales como: aspectos centrales previo a la realización de la evaluación Ex-post del proyecto de la entidad ejecutora, se aborda una revisión del marco conceptual y metodológico de la guía de evaluación Expost de proyectos de riego, $y$ específicamente se utilizara la herramienta de la guía de evaluación Ex-post de proyectos de riego para entidad ejecutora del proyecto, como documento de trabajo los cuales serán descritos de una manera clara y precisa en el desarrollo de la investigación.
La investigación que se presenta permitirá conocer los problemas críticos respecto de los resultados del Proyecto de Inversión Pública, para tratar de coadyuvar con el desarrollo del país, aún se desconoce el efecto real de cada uno de los proyectos que han sido ejecutados y que se encuentran en pleno funcionamiento.

La guía de evaluación ex-post de proyectos de riego, se constituye en una herramienta que permite la comparación de lo planificado con lo realizado, el mismo servirá de orientación para los tomadores de decisiones de los ejecutores de proyectos de riego para una mejor programación de los mismos. (Vera, 1997).

La evaluación Ex-post de proyectos de riego es especialmente importante en los países en desarrollo, donde los recursos son escasos y lo gastado debe maximizar su efecto en la reducción de la pobreza. Si los proyectos están mal diseñados, no llegan a los beneficiarios previstos o despilfarran los recursos, con la información adecuada es posible rediseñarlos, mejorarlos si se estima necesarios. El conocimiento obtenido de los estudios para evaluar los efectos también proporcionara información decisiva para el 
diseño adecuado de futuros programas y proyectos.

Considerando al Proyecto Represa y Sistema de Riego Cuartos por sus objetivos como proyecto de desarrollo rural, la evaluación ex- post de este proyecto se realiza con la finalidad de valorar el logro de los resultados generales en términos de eficiencia, producto efecto e impacto $\mathrm{y}$ obtener enseñanzas aplicables en las actividades de desarrollo rural a realizarse a futuro.

Conocer el efecto e impacto del Proyecto Represa y Sistema de Riego Cuartos permitirá no solo cuantificar los resultados obtenidos sino también tomar conciencia de la necesidad de realizar la evaluación ex-post en el corto, mediano o largo plazo.

El propósito del estudio radica en realizar la evaluación Ex- Post del proyecto "Construcción Sistema de Riego Cuartos", para medir el grado de cumplimiento de los objetivos del proyecto, comparando los objetivos propuestos con los objetivos alcanzados, mediante la guía de evaluación Ex- Post propuesto por el Ministerio de Medio Ambiente y Aguas.

La aplicación de la guía de la evaluación Ex-post de proyectos de riego, permitirá determinar si el proyecto ha sido eficaz y eficiente en su implementación, alcanzando los objetivos, metas y resultados propuestos, los mismos que se reflejan en la pertinencia de su implementación, sostenibilidad e impactos que los mismos se proponían lograr en la población y el proyecto, particularmente en el proyecto "Construcción Represa y Sistema de Riego Cuartos".

METODOLOGÍA

El estudio se enmarcó bajo el tipo de investigación cuantitativo con alcance descriptivo, se adoptó ese razonamiento para reunir la información necesaria y pertinente en beneficio de los objetivos. Para determinar los resultados alcanzados por el proyecto y su efecto en la población beneficiaria respecto a su pertinencia, sostenibilidad e impacto generado en el bienestar de la población beneficiaria, el enfoque para la evaluación del proyecto ha sido mediante su marco lógico, el cual permite determinar, cuantificar y comparar los objetivos previstos con los alcanzados y/o logrados y la variación de la situación inicial (Ex ante) respecto a la situación actual (Ex-post).

Para la ejecución de la evaluación Expost de un proyecto de riego se comprende la realización de varias actividades, las cuales se las puede agrupar en tres: 1. Actividades prepar atorias, 2. Realización de la evaluación de resultados y 3. Retroalimentación de la evaluación de resultados.

La zona donde se llevó a cabo el estudio fue en la región Sur, Departamento Potosí, Provincia, Modesto Omiste en la Comunidad Cuartos, Geográficamente ubicada en el área del proyecto, se inscribe entre las siguientes coordenadas: Latitud Sur:

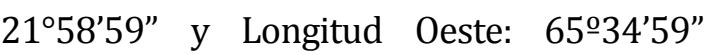
Altura: 3414 m.s.n.m.

Además, como método de análisis se gestionó ante la entidad ejecutora del proyecto que se proporcionaran el estudio Testudio Económico Social (Social, 1993) y Ambiental (TESA) elaborado en la gestión 2009, además 10 Planillas de Pago, Contrato Modificatorio, Libro de Órdenes y los Planos Asbuit del proyecto ejecutado desde el 11 de julio del 2014 hasta el 3 de Marzo del 2016, estos documentos se proporcionaron en calidad de préstamo de los archivos de la Dirección de Riego dependiente de la 
Secretaria de Desarrollo Agropecuario y Seguridad Alimentaria del Gobierno Autónomo Departamental de Potosí, para su revisión en la biblioteca de la misma Dirección de Riego.

En este punto se describieron en detalle los datos que se requieren para la evaluación Ex-post de productos y efectos, y se propusieron técnicas de recolección y métodos de análisis que fueron usadas en el trabajo de campo y gabinete mediante el uso de fichas (formularios). Hay que considerar que las técnicas y métodos propuestos no siempre son aplicables, por lo que en algunos casos el evaluador debe recurrir a su propia experiencia y creatividad para obtener los datos requeridos.

\section{Materiales}

El material necesario para realizar la evaluación Ex-post del sistema de riego se detalla a continuación:

\section{Equipos utilizados en campo}

Los equipos que se utilizaron para las diferentes salidas de campo fueron: Brújula, GPS GARMIN; Cámara fotográfica; Libreta de campo, Fichas de campo para recabar información, entre otros.

\section{Equipo de procesamiento e impresión}

En el equipo que se utilizó para el procesamiento de la información, elaboración de planos, gráficos, redacción e impresión fue: Laptop hp Core I7" de 7ma generación Impresora EPSON L380, Memoria USB ( 2 Teras), Software: AutoCAD 2015, Microsoft Excel 2016, Microsoft Word 2016 Microsoft PowerPoint 2016 ABRO 0.2 ver 3.1, Google Earth.

\section{Materiales de gabinete}

Estudio a diseño final T.E.S.A., Expediente Técnico de ejecución de proyecto Planillas de avance del proyecto, Planilla de cierre del proyecto Libro de Órdenes, Padrón de usuarios de beneficiados actualizado. Papel tamaño carta para la impresión. Plumones, resaltadores, lapiceros y lápices.

\section{Recursos humanos}

Tesista, Presidente del comité de obras Chofer de Camioneta

\section{RESULTADOS Y DISCUSIÓN}

Para realizar la evaluación Ex-post de productos se usó de cuatro fichas detalladas a continuación:

\section{Ficha 1}

La FICHA 1 contiene la COMPARACIÓN CUANTITATIVA DE PRODUCTOS PREVISTOS Y EJECUTADOS. Los datos correspondientes a los productos son el tipo y número de obras hidráulicas construidas, diferenciando las obras principales. En la ficha se detallaron las metas formuladas en el diseño con las cuales se aprobó el financiamiento, y se verificaron si efectivamente fueron alcanzadas a través de la revisión del libro de órdenes, informes de supervisión y planos las built, como también mediante la visita de campo. Se recomienda incorporar detalles acerca de las características de las estructuras propuestas y ejecutadas. Los datos deben ser apoyados con fotos.

Por otro lado, en cuanto al servicio de Acompañamiento/Asistencia Técnica contenido en la FICHA 1, solo se verificó el diseño final la necesidad de este servicio y si éste fue ejecutado efectivamente durante la construcción de obras y la puesta en 
operación del proyecto. Asimismo, se verificó el número de familias a las cuales se propuso el apoyo participación efectiva en el proyecto.

Respecto al análisis del área de riego contenido en la FICHA 1, verificó si el perímetro de influencia había variado como efecto de la intervención del proyecto. Lo más práctico fue estimar el área mediante una foto aérea antes de la visita de campo, para identificar los límites de la zona de riego. En casos excepcionales existen planos parcelarios que permitieron hacer una mejor estimación. En caso de no contar con fotos ni con planos, se recorrió el perímetro del área de influencia con un GPS para una estimación rápida de esta superficie.

\section{Ficha 2}

La FICHA 2 contiene el resumen del análisis sobre la CALIDAD DE LOS PRODUCTOS EJECUTADOS. Esta ficha fue aplicada para analizar y comparar la calidad y durabilidad de las diferentes obras, con especial atención de las obras cuya operatividad sostiene total o parcialmente el sistema de riego (por ejemplo la obra de toma o los acueductos y sifones en el canal principal). La comparación se realizó entre los resultados reales y actuales con las especificaciones técnicas esperadas que fueron establecidas en el diseño final del proyecto.

Debido a que el análisis se realizó por estructura, se propuso obtener conclusiones por obra $y$, posteriormente, sacar conclusiones acerca de su efecto para el sistema en su conjunto. Durante este proceso no hay que perder de vista que se pretende también verificar si existen condiciones de sostenibilidad del sistema, las cuales pueden darse para todo el sistema o bien solo para una parte.
La información recolectada en la FICHA 2 permitió obtener conclusiones sobre la operatividad de la obra, el grado de requerimiento de mantenimiento, el costo estimado de reparaciones o adecuaciones necesarias y la existencia de riesgo de colapso.

\section{Ficha 3}

La FICHA 3 contiene la COMPARACIÓN ENTRE COSTO PROPUESTO Y COSTO REAL EJECUTADO POR RESULTADO (ejecución de obras y servicios) y por tipo de estructura (embalse, captación, conducción y distribución). Considerando los costos por productos incurridos en las diferentes etapas del proyecto, se logró establecer la EFICIENCIA DE COSTOS DEL PROYECTO.

Para determinar los costos presupuestados se usó información proveniente del documento del diseño final con el cual se aprobó el financiamiento del proyecto. Por otro lado, para determinar los costos reales se recurrió a información proveniente de las planillas de pago.

\section{Ficha 4}

La FICHA 4 contiene la COMPARACIÓN ENTRE TIEMPOS PROPUESTOS Y TIEMPOS REALES DE EJECUCIÓN POR RESULTADO (obras y servicios) y por tipo de estructura (embalse, captación, conducción y distribución) para establecer LA EFICIENCIA DE TIEMPOS DEL PROYECTO.

Finalmente, en el análisis de resultados fueron considerados los problemas que se abordaron durante la ejecución del proyecto. Entre éstos se encontraron los referidos a la calidad de los estudios de pre-inversión, la administración del proyecto, modalidad de ejecución, disponibilidad de recursos técnicos, financieros y humanos de la entidad ejecutora, disponibilidad de 
materiales de construcción, disponibilidad de contratistas capacitados y condiciones externas (clima, desastres naturales, conflictos sociales, entre otros).

\section{Análisis de los efectos}

Para la evaluación Ex - Post de efectos fueron usadas nueve fichas (formularios) detallados a continuación:
AUTOGESTIÓN DEL SISTEMA DE RIEGO basado en los requerimientos de funcionamiento u operación previstos y actuales del sistema de riego. Los mismos deberán verificarse a través de tareas mínimas a cumplir por los usuarios a cargo de la operación y mantenimiento de la infraestructura construida presentada en la tabla 3 como se muestra a continuación:

\section{Ficha 5}

La FICHA 5 contiene el análisis de CAMBIOS EN LAS CONDICIONES PARA LA

Tabla 3. Tareas mínimas de operación y mantenimiento de sistemas de riego

\begin{tabular}{ll}
\hline \multicolumn{1}{c}{ Tipo de tareas } & \multicolumn{1}{c}{ Descripción } \\
\hline Constitucionales & $\begin{array}{l}\text { Composición del grupo de usuarios, derechos y } \\
\text { obligaciones. } \\
\text { Actividades de distribución de agua, operación de } \\
\text { infraestructura, mantenimiento. }\end{array}$ \\
Organización interna & $\begin{array}{l}\text { Toma de decisiones colectivas, cargos en la } \\
\text { organización, comunicación entre los socios. }\end{array}$ \\
Movilización de recursos & $\begin{array}{l}\text { Trabajos colectivos (con aporte de mano de obra), recaudación } \\
\text { de aportes en dinero. }\end{array}$ \\
Reconstrucción de obras & Capacidad de organización para la reconstrucción de obras. \\
Relacionamiento externo & Con ONGs, municipio, proveedores.
\end{tabular}

Tareas mínimas de operación y mantenimiento (Fuente: MMAYA 2014)

Las primeras cuatro tareas fueron fundamentales para sustentar la autogestión de un sistema de riego, por lo que fue necesario el análisis de todos los años del funcionamiento del sistema de riego. Las últimas dos tareas fueron coyunturales: la capacidad de reconstrucción de obras solo para el caso de que se necesitara reconstruir una obra; y el relacionamiento externo solo en caso de requerirse apoyo para la reconstrucción.

Para verificar la información, respecto a la autogestión del sistema de riego, se recurrió a las siguientes fuentes de información: documentos del proyecto, documentos de acompañamiento, libro de actas, entrevistas y visita de campo. La verificación cruzada de datos debe llevar a conclusiones certeras. La evaluación Expost de las condiciones de autogestión concluyó con preguntas sobre la posible existencia de amenazas externas o internas, que podían poner en riesgo la continuidad de la organización de los regantes. 


\section{Ficha 6}

La FICHA 6 permite establecer el INCREMENTO EN LA DISPONIBILIDAD DE AGUA basado en una hoja electrónica de cálculo. Para la aplicación de la misma se recolectaron datos de disponibilidad de agua sin y con proyecto. Los datos de la situación sin proyecto se obtendrían del diseño final y los datos de la situación con proyecto se determinaron en la visita de campo.

En el caso de embalses, una vez introducidos los datos de campo en la hoja electrónica, éstos fueron convertidos en volúmenes de agua disponibles en los períodos de los cultivos bajo los siguientes supuestos:

El volumen de agua embalsada fue dividido proporcionalmente entre los meses de largadas. Durante el período de largadas, la pérdida por evaporación en el embalse fue cero.

La eficiencia de conducción entre la presa y la obra de toma es la misma en la situación sin y con proyecto, por lo que se utilizaron los datos del diseño final.

A cada material de construcción se aplicó un factor de eficiencia de conducción por kilómetro, basado en la sistematización de estudios existentes sobre el tema. La eficiencia total se consiguió con la fórmula: $\mathrm{Ef}$ total $=(\mathrm{Ef}$ por $\mathrm{km}$ ) x longitud.

\section{Ficha 7}

La FICHA 7 analizó los INCREMENTOS EN LA PRODUCCIÓN AGROPECUARIA, basada en que una mayor oferta de agua en el proyecto se traduce en cambios en la superficie cultivada, la cédula de cultivos, calendario agrícola y rendimientos.
Para el análisis de esta variable se consideraron dos niveles de recolección y análisis de información: uno es el nivel de los cambios en la producción familiar y - el otro nivel - representa una extrapolación de dicha información para toda el área regada.

La experiencia mostró que resulta difícil obtener información general (sobre el total del área de influencia del sistema) a través de entrevistas colectivas 0 individuales. Usualmente, en una entrevista, un agricultor o dirigente responde en base al conocimiento que tiene sobre su propio predio.

Los datos de las cédulas de cultivo de las familias están reflejados en la FICHA 7: INCREMENTO EN LA PRODUCCIÓN AGRÍCOLA - encuesta familiar, con la que se recolectaron información sobre la cédula de cultivo sin y con proyecto. A los agricultores se les solicitó su opinión con respecto a los cambios a consecuencia del proyecto, con relación al tipo de cultivos, composición de la cédula, épocas de cultivo, área regada y rendimiento de los cultivos. Asimismo, en la FICHA 7 se resumen los datos de las encuestas familiares para determinar conclusiones sobre los principales cambios.

Finalmente, debe hacerse hincapié en que la recolección de datos y el análisis de cambios se realizaron el primer día de la visita de campo. Estos datos fueron inmediatamente analizados para inferir sobre la cédula del área total del sistema y concluir sobre los cambios principales a causa del proyecto. En cambio, en el segundo día de la visita de campo, se levantaron datos de producción de los cultivos que fueron señalados como 
sujetos de cambio. De estos cultivos, debe realizarse una investigación más exhaustiva en cuanto a costos de producción y rendimiento. La información de los cambios en la producción es esencial para el análisis económico del proyecto.

\section{Ficha 8}

En la FICHA 8 se estableció el INCREMENTO EN EL ÁREA BAJO RIEGO ÓPTIMO (ABRO) por efecto del proyecto. Los datos de las cédulas de cultivo "sin proyecto", y en la situación real verificada en campo "con proyecto" se introdujeron en el software ABRO del Viceministerio de Recursos Hídricos y Riego, para determinar el área incremental bajo riego óptimo resultante del proyecto. A modo de ejemplo se presenta en anexos la primera hoja de salida del ABRO en la investigación original, sin embargo, las 9 hojas de salida fueron incluidas en el informe de evaluación.

\section{Ficha 9}

La FICHA 9 contiene el análisis sobre los CAMBIOS EN LA APLICACIÓN DE AGUA EN LAS PARCELAS, mediante la verificación de cambios en el método y la calidad de la aplicación de agua en las parcelas, reflejada en la tecnología de manejo de agua en la parcela. La calidad de la aplicación de agua en las parcelas se entiende como el grado de satisfacción de los requerimientos de los cultivos, en términos de oportunidad, uniformidad y cantidad de agua aplicada.

La verificación de los métodos de riego se hizo por medio de la observación en el campo, apoyada por entrevistas con dirigentes. Adicionalmente, fueron identificados los distintos métodos de riego (aspersión, goteo, gravedad, entre otros) y su cobertura, incluyendo el estado actual y los cambios en la tecnología de manejo del agua en la parcela. Para ello, se debe observar e indagar sobre los criterios y conocimientos que los agricultores manejan sobre las variaciones en la aplicación del agua, así como la observación en campo del grado de control sobre el flujo del agua en la parcela, y la existencia o ausencia de pérdidas por escurrimiento.

\section{Ficha 10}

La evaluación de los efectos económicos se inició con la recolección de datos de todos los cultivos mediante la FICHA 10. DATOS AGROECONÓMICOS TODOS LOS CULTIVOS. Los datos de esta ficha son de carácter general, basados en una entrevista con dos o tres agricultores respecto a los datos económicos de la situación sin proyecto y los de la situación con proyecto.

Para simplificar la evaluación se indagó sobre datos económicos detallados solo para los cultivos nuevos y los que según los entrevistados sufrieron cambios sustanciales en sus rendimientos, fue incluida esta información en la FICHA 10A: COSTOS E INGRESOS DE PRODUCCIÓN POR HECTÁREA - CULTIVOS CON CAMBIOS. Para los demás cultivos fueron asumidos los rendimientos y las cantidades de insumos que se mantuvieron, por lo que solo fueron actualizados los precios de los insumos y de los productos con relación a la situación sin proyecto.

Seguidamente, una vez ya recopilados los datos mencionados anteriormente fueron introducidos en las Planillas Parametrizadas del VIPFE. Para esta evaluación se tomó en cuenta la situación sin proyecto, en la cual se analizarán los beneficios que generaron los productores 
tomando en cuenta los costos de producción y los ingresos de la cédula de cultivos antes de la ejecución del proyecto, generando un flujo de fondos sin proyecto. Posteriormente, se analizó el flujo de fondos con proyecto, incorporando la cédula de cultivos nueva. Después se determinó el flujo de costos y de ingresos incrementales del proyecto para un período de diez años o el horizonte de vida de cada proyecto, preparando las planillas correspondientes, y se calcularon los indicadores económicos del proyecto (Valor Actual Neto Privado y Social, Valor Actual de Costos, Tasa Interna de Retorno, Relación Beneficio/Costo).

Finalmente, comparando los indicadores económicos antes y después del proyecto, se pudo determinar los efectos económicos de la inversión.

\section{Ficha 11}

La FICHA 11 acerca de CAMBIOS EN LA DISTRIBUCIÓN SOCIAL DE LOS BENEFICIOS DE LA INVERSIÓN sirvió para verificar el beneficio obtenido con el proyecto en partes de agua (expresado en turnos, horas por turnos y/o superficie regada, de acuerdo con las características de cada proyecto). Sobre la base de estos datos, se hizo un cálculo de la distribución social de los beneficios.

Asimismo, la FICHA 11 permitió indagar el efecto del proyecto en las relaciones de género, verificando si el proyecto tuvo acciones específicas para mujeres y produjo impactos específicos en ellas. Para este fin se buscaron datos sobre número de familias encabezadas por mujeres con derechos de agua y participación de mujeres en la organización de riego, a través de las entrevistas.

En la parte final de la FICHA 11 se presentó la comparación de costos - eficiencia por unidad de beneficio, es decir: costo por hectárea regable, costo por hectárea regada, costo por hectárea incremental ABRO, costo por familia beneficiaria y costo por $1.000 \mathrm{~m} 3$ de agua producido, utilizando datos de costos del diseño final y de las planillas de pago para los costos reales.

\section{Ficha 12}

La FICHA 12 sobre la EVALUACIÓN DE RIESGOS AMBIENTALES, permitió identificar y prevenir los riesgos ambientales que podrían afectar la sostenibilidad del sistema. Se concluyó sobre el nivel de riesgo de colapso del sistema a partir de los riesgos ambientales, además los siguientes: a). Afectación del ecosistema en caso de construcción de embalses. b). Inestabilidad de los terrenos en los que se ubican las obras; y c). Degradación de los terrenos cultivables.

\section{Ficha 13}

Finalmente, la FICHA 13 se plantaron las CONDICIONES PARA LA SOSTENIBILIDAD, la cual permitió concluir sobre la sostenibilidad del sistema de riego evaluado mediante la verificación de las siguientes condiciones mínimas de sostenibilidad en el sistema: La calidad y durabilidad de las obras (FICHA 2). Las condiciones de autogestión (FICHA 5). La ausencia de riesgos ambientales (FICHA 12).

\section{Evaluación expost (interpretación)}

El proceso de evaluación Ex-post concluye con un dictamen sobre el logro de los objetivos del proyecto, de modo tal, que se puede concluir si el proyecto es eficiente, eficaz, impactante, sostenible y pertinente.

Un proyecto es calificado como eficiente cuando ha generado los productos 
(asociados a los componentes) esperados, con igual calidad y con la misma o menor cantidad de recursos programados y en un tiempo igual o menor al previsto.

Un proyecto es calificado como eficaz cuando el objetivo central del proyecto ha sido alcanzado. La eficacia global del proyecto se juzga considerando las condiciones de operación y utilización de los productos generados en la fase de inversión, la rentabilidad social en las condiciones reales y el hecho de haber logrado el objetivo central del proyecto.

Un proyecto es calificado como impactante cuando produce efectos directos intencionales favorables sobre los beneficiarios del proyecto, así como sobre las condiciones socioeconómicas y ambientales del ámbito de influencia del proyecto.

Un proyecto es calificado como sostenible cuando los productos generados en la fase de ejecución del proyecto tienen gran probabilidad de ser operados $\mathrm{y}$ utilizados durante la vida útil del proyecto.

Un proyecto es evaluado como pertinente cuando ha sido planificado y ejecutado de acuerdo con la política sectorial-funcional, el contexto regional y local, y responde a las necesidades de los beneficiarios.

Para posibilitar la formulación de las conclusiones y dictamen de la evaluación Expost, se utilizará la tabla sobre la "Calificación sobre el logro de los diferentes criterios e indicadores" la cual se encuentra en la investigación original, la cual permitió verificar el logro de los diferentes indicadores de evaluación con el sustento de los datos comparados entre la situación sin y con proyecto que están contenidos en las diferentes FICHAS.

Los resultados se describen de acuerdo a los objetivos de la investigación que son:

\section{a) Aplicar la guía de evaluación ex- post propuesta por el Ministerio de Medio Ambiente y Aguas.}

Para esta pregunta se realizó el llenado de 13 fichas, del cual se pudo recabar la siguiente información que se muestra en la Tabla 4.

Tabla 4. Cuadro de Fichas de evaluación ex - post de proyectos de riego

\begin{tabular}{ll}
\hline \multicolumn{1}{c}{ No de Ficha } & \\
\hline Tabla 1 & Calificación sobre el logro de los diferentes criterios e indicadores \\
Ficha 1 & Comparación cuantitativa de productos o componentes del proyecto \\
Ficha 2 & Calidad de las obras \\
Ficha 2-A & Calidad de la obra después de la ejecución del proyecto \\
Ficha 2-B & Calidad de la obra de captación después de la ejecución del proyecto \\
Ficha 2-C & Calidad de la presa después de la ejecución del proyecto \\
\hline
\end{tabular}




\begin{tabular}{ll}
\hline No de Ficha & Descripción \\
\hline
\end{tabular}

Ficha 2-D Calidad de acueductos y puentes colgantes después de la ejecución del proyecto

Ficha 2-E Calidad de sifones después de la ejecución del proyecto

Ficha 2-F Calidad de los estanques y atajados después de la ejecución del proyecto

Ficha 2-G Calidad de rápidas después de la ejecución del proyecto

Ficha 3 Eficiencia en el costo del proyecto

Ficha $4 \quad$ Eficiencia en el tiempo de la ejecución del proyecto

Ficha 5 Cambios en las condiciones para la autogestión del sistema de riego

Ficha 6 Incremento en la disponibilidad de agua

Ficha 7 Incremento en la producción agrícola

Ficha 8 Balance hídrico y cálculo de áreas incrementales

Ficha 9 Cambios en la aplicación del agua en las parcelas

Ficha 10 Datos económicos de la producción - todos los cultivos

Ficha 10-A Costos de producción - cultivos con cambios

Ficha 11 Cambios en la distribución de beneficios sociales de la inversión

Ficha 12 Evaluación de riegos ambientales

Ficha 13 Evaluación de la sostenibilidad

Fichas de evaluación Ex - Post (Fuente: MMAYA 2014)

b) Determinar la Pertinencia, Eficiencia, Eficacia, Impacto y Sostenibilidad del proyecto Construcción Represa y Sistema de Riego Cuartos.

Para esta pregunta también se realizó el llenado en base a la evaluación ex - post que indica las fichas de evacuación, que representa el resumen de la evaluación Ex - Post, del cual se pudo recabar la siguiente información que se muestra en la Tabla 5. 
Tabla 5. Calificación sobre el logro de los diferentes criterios e indicadores

\begin{tabular}{|c|c|c|c|c|}
\hline \multirow{2}{*}{ CRITERIOS } & \multirow{2}{*}{ INDICADORES } & \multicolumn{3}{|c|}{$\begin{array}{c}\text { Fuente de LOGRO / } \\
\text { Información CUMPLIMIENTO }\end{array}$} \\
\hline & & № FICHA & SI & NO N/A \\
\hline \multirow{5}{*}{ EFICIENCIA } & Nivel de ejecución de componentes & 1 у 2 & SI & \\
\hline & Eficiencia en el costo & 3 & & NO \\
\hline & Eficiencia en el tiempo de ejecución & 4 & & NO \\
\hline & $\begin{array}{l}\text { Problemas, limitaciones y debilidades en la } \\
\text { ejecución }\end{array}$ & & SI & \\
\hline & Eficiencia Global & & SI & \\
\hline \multirow[t]{8}{*}{ EFICACIA } & $\begin{array}{l}\text { Disponibilidad de los productos (capacidad) } \\
\text { Incremento en la disponibilidad de agua } \\
\text { № de la que se pueden regar (área regable). }\end{array}$ & 6 y 8 & SI & \\
\hline & $\begin{array}{l}\text { Utilización de los bienes y servicios } \\
\text { Cambios en las condiciones para la } \\
\text { autogestión del sistema de riego }\end{array}$ & 5 & SI & \\
\hline & $\begin{array}{l}\text { Cambios en la demanda anual de agua para } \\
\text { riego de X m3. }\end{array}$ & 8 & SI & \\
\hline & $\begin{array}{l}\text { Cambios en la aplicación del agua en la } \\
\text { parcela }\end{array}$ & 9 & & NO \\
\hline & $\begin{array}{l}\text { Beneficios Directos Intencionales Incremento } \\
\text { en el volumen de producción agrícola TM/año. }\end{array}$ & . & SI & \\
\hline & $\begin{array}{l}\text { Cambios en la rentabilidad económica- social. } \\
\text { Indicador } \\
\text { VANS ex ante/ VANS Ex-post }\end{array}$ & 10 & SI & \\
\hline & $\begin{array}{l}\text { Cambios en la distribución social de los } \\
\text { beneficios del proyecto }\end{array}$ & 11 & SI & \\
\hline & Eficacia global & & SI & \\
\hline
\end{tabular}




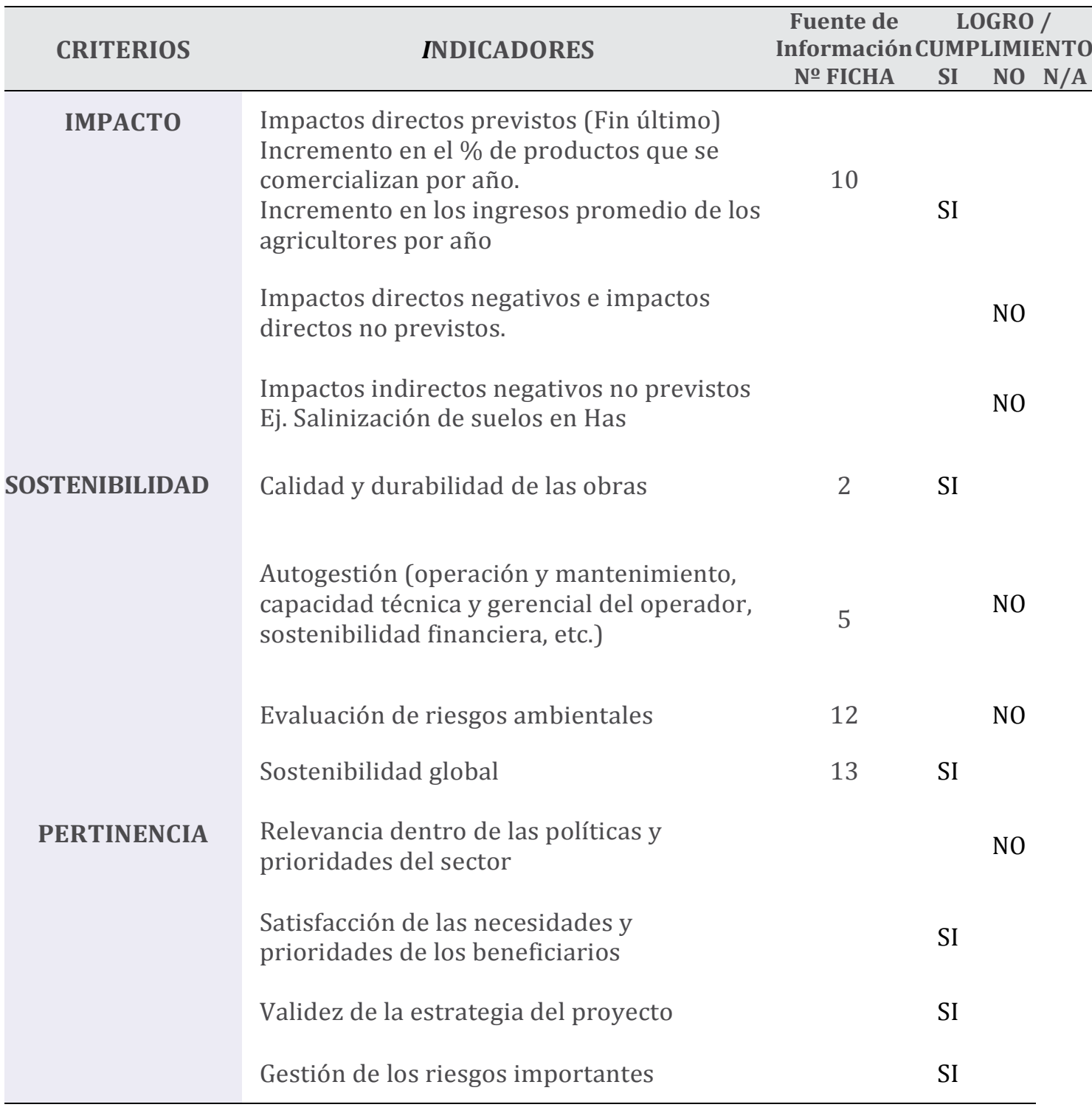

Calificación de evaluación Ex - Post (Fuente: MMAYA 2014)

\section{c) Determinar la avaluación ex - post de resultados y la evaluación ex - post de efectos del proyecto Construcción Represa y Sistema de Riego Cuartos.}

Para esta pregunta se realizó la cuantificación porcentual de los criterios de la evaluación ex - post, cumplidos después de la ejecución de proyecto, del cual se pudo recabar la siguiente información que se muestra en la Tabla 6. 
Tabla 6. Parámetros de Calificación

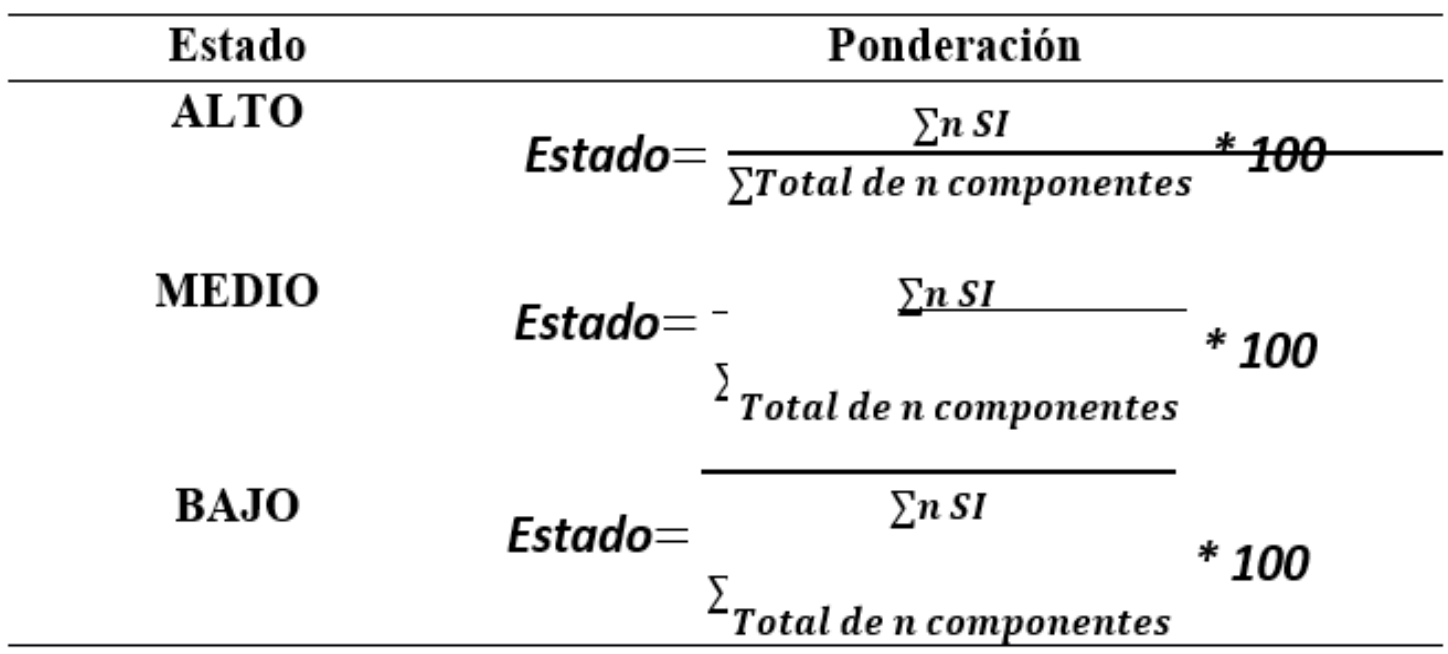

Parámetros de calificación (Fuente: elaboración propia)

Tabla 7. Cumplimiento del proyecto ejecutado con relación al proyecto previsto

\begin{tabular}{lc}
\hline CRITERIOS & \% DE CUMPLIMIENTO \\
\hline EFICIENCIA & $60 \%$ \\
EFICACIA & $88 \%$ \\
IMPACTO & $33 \%$ \\
SOSTENIBILIDAD & $50 \%$ \\
PERTINENCIA & $75 \%$ \\
TOTAL $=$ & $\mathbf{6 1 \%}$ \\
\hline
\end{tabular}

Cumplimiento del proyecto ejecutado a lo previsto (Fuente: elaboración propia)

En la Tabla 7 anterior indica que la evaluación cumple con un $\mathbf{6 1 \%}$ de ejecución del proyecto programado al proyecto previsto.

El análisis y ponderación de variables e indicadores se realizó por el método de la evaluación Ex - Post propuesto por el Ministerio de Medio ambiente y Aguas aplicado al proyecto Construcción Represa y Sistema de Riego Cuartos, tomando en cuenta la siguiente calificación: $(\mathrm{BAJO})=35 \%, 36=$ $($ MEDIO $)=70 \%, 71=($ ALTO $)=100 \%$
- ALT0.- Resultados que sobresalen respecto de lo esperado.

- MEDIO.- Resultados similares a lo esperado.

- BAJ0.- Resultado por debajo de lo esperado.

Por lo tanto el porcentaje de cumplimiento del $\mathbf{6 1 \%}$ está en el rango de Medio por lo que los resultados del proyecto son similares a los resultados esperados. 
Evaluación ex - post de Resultados del Proyecto Construcción Represa y Sistema de Riego Cuartos

Tabla 8. Evaluación ex - post de resultados

\begin{tabular}{lc}
\hline Criterios & Evaluación Ex - Post de Resultados \\
\hline PERTINENCIA & $75 \%$ \\
EFICIENCIA & $60 \%$ \\
EFICACIA & $88 \%$ \\
IMPACTO & $33 \%$ \\
SOSTENIBILIDAD & $50 \%$ \\
TOTAL $=$ & $\mathbf{6 1 \%}$ \\
\hline
\end{tabular}

Evaluación ex - post de resultados (Fuente: elaboración propia)

\section{CONCLUSIONES}

La aplicación de la guía de evaluación Ex - Post estableció que el proyecto se implementó con una eficacia del $88 \%$, una eficiencia del $60 \%$, una pertinencia del $75 \%$, una sostenibilidad del $50 \%$ y tiene un impacto del 33\%, con base en los resultados obtenidos en la investigación.

La evaluación ex - post no es sinónimo de control o fiscalización, se trata de analizar los resultados de los proyectos y obtener lecciones aprendidas para mejorar la calidad de la inversión pública, así como proveer de información a las entidades ejecutoras de proyectos de inversión.

En Bolivia, la evaluación ex-post ha tenido una escasa difusión a pesar de la existencia de una guía de evaluación de proyectos ex - post proporcionado por el Ministerio de Medio Ambiente y Aguas.

La organización de los beneficiarios del proyecto es deficiente pero se están fortaleciendo poco a poco, se están tomando decisiones de manera democrática y en resoluciones de la Asamblea General para cualquier acción.

La eficiencia del proyecto alcanzo $60 \%$ por que los resultados fueron similares a los esperados, la eficacia alcanzo el $88 \%$ por los que los resultados sobresalen a los esperados, el impacto alcanzo 33\% y que los resultados están por debajo a los esperados, la sostenibilidad alcanzo el $50 \%$ los resultados fueron similares a los esperados y la pertinencia alcanzo el 75\% los resultados sobresalen a los esperados.

La evaluación ex - post de resultados alcanzo el $61 \%$ y los resultados obtenidos son Medios y son similares a los esperados del proyecto propuesto. La evaluación ex post de efectos alcanzo el $42 \%$ y los resultados obtenidos son Medios y son similares a los esperados del proyecto propuesto.

En general el proyecto tiene un alcance MEDIO, se dice que los resultados de la evaluación tienen una respuesta, en la cual lo beneficiarios manifestaron que el proyecto aún no ha colmado o abarcado todas las expectativas esperadas. Aun el proyecto no cumple con las metas establecidas.

De acuerdo al análisis de los resultados medios obtenidos se puede establecer que son debido a que el proyecto en la etapa de construcción no tubo Acompañamiento, no tuvo Asistencia Técnica en Riego durante la fase de puesta 
en marcha y no está contemplado la Asistencia Técnica en Producción Agrícola con Riego de los regantes.

\section{REFERENCIAS}

Almendras, J. A. (2016). Supervisión y fortalecimiento Ex-post proyectos de riego A/AT mi agua III. Potosí

CAF, B. (2014). Notas de evaluación EX POST. CAF

Gobierno Autónomo Departamental De Potosí. (2016). Construcción represa y sistema de riego cuartos. Potosí

MMAYA. (2012). Proyecto Ley Marco Agua para La Vida. La Paz - Bolivia: Estado Plurinacional de Bolivia

MMAYA. (2013). Inventario Nacional de Sistemas de Riego. La Paz: Programa de Desarrollo Agropecuario Sustentable (PROAGRO)

MMAYA. (2014). Guía para la Evaluación Expost de Proyectos de Riego. La Paz: Programa de Desarrollo Agropecuario Sustentable (PROAGRO)
Montaño, C. A., Rodríguez, E., Panche, P., \& Sosa, Y. (s.f.). SLIDESHARE. Obtenido de https://es.slideshare.net/juliomabeljaqu ecarmenza/evaluacion-ex-postpresentacion

RIEGO, V. D. (2 de 6 de 2015). Mi riego más inversión para riego. Obtenido de http://www.miriego.gob.bo/component es.php?Seleccion=21

SINNAPS. (S.F.). DEFINICION DE PROYECTO. Obtenido de https://www.sinnaps.com/blog-gestionproyectos/definicion-de-proyecto

Social, I. L. (1993). Propuesta Metodológica para La Evaluación Ex-post y El Informe de Término de los Proyectos de Inversión. Santiago: Chile LC/IP/L 84

Vera, P. (1997). Guía Metodológica para la Evaluación Ex-Post de Proyectos. Santiago, Chile: Instituto Latinoamericano y del Caribe de Planificación Económica y Social - ILPES 